$+F_{Z}^{p}(X, A)$. The reverse inclusion is clear and the equality shows that $\tau$ is onto.

\title{
REFERENCES
}

1. S. Lefschetz, Algebraic topology, Amer. Math. Soc. Colloquium Publications, vol. 27, New York, 1942.

2. E. H. Spanier, Cohomology theory for general spaces, Ann. of Math. vol. 49 (1948) pp. 405-427.

TULANE UNIVERSITY

\section{A NOTE ON A COMBINATORIAL PROBLEM}

\section{H. J. RYSER}

The purpose of this note is to prove the following theorem.

TheOREM. Let $x_{1}, x_{2}, \cdots, x_{v}$ denote $v$ distinct elements and let $T_{1}, T_{2}, \cdots, T_{v}$ denote $v$ sets formed from these elements. Suppose further that each $T_{i}$ consists of exactly $k$ distinct elements and that every pair of distinct sets $T_{i}$ and $T_{j}$ have exactly $\lambda$ elements in common $(0<\lambda<k<v)$. Then

$$
\lambda=\frac{k(k-1)}{v-1} .
$$

The combinatorial problem under consideration makes its appearance in the study of projective planes, Hadamard matrices, and block designs. In the combinatorial problem of Todd arising in the study of Hadamard matrices it is usually assumed that $\lambda$ $=k(k-1) /(v-1)[3 ; 4] .{ }^{1}$ For the symmetrical block designs each element of the arrangement is required to occur exactly $k$ times, and it is then easy to verify that $\lambda=k(k-1) /(v-1)[1]$. Further theorems concerning the possibility of this combinatorial problem for a given $v, k$, and $\lambda$ may be found in [3].

To prove the theorem let the elements $x_{1}, \cdots, x_{v}$ be listed in a row, and let the sets $T_{1}, \cdots, T_{v}$ be listed in a column. Form the incidence matrix $A$ of the arrangement in the usual way by inserting a one in column $i$ and row $j$ if $x_{i}$ belongs to set $T_{j}$, and a zero in the contrary case $[2 ; 3]$. Now let $s_{i}$ denote the sum of column $i$ of the

Presented to the Society, September 2, 1949; received by the editors June 9, 1949.

${ }^{1}$ Numbers in brackets refer to the references cited at the end of the paper. 
matrix $A$. Let row $r$ of $A$ have ones in the $k$ columns $r_{1}<r_{2}<\cdots<r_{k}$, and let $t=\lambda(v-1)+k$. Then by the hypothesis of the theorem it follows that

$$
s_{r_{1}}+s_{r_{2}}+\cdots+s_{r_{k}}-t=0 .
$$

The matrix of the above system of $v$ equations in $v$ unknowns is simply $A$. Moreover, it is clear that

$$
s_{1}+s_{2}+\cdots+s_{v}-k v=0 .
$$

Now $t \neq 0$, and consequently the matrix

$$
C=\left[\begin{array}{cc} 
& 1 \\
A & \vdots \\
& i \\
1 \cdots 1 & k v / t
\end{array}\right]
$$

has a zero determinant. Multiplying $C$ by its transpose implies that

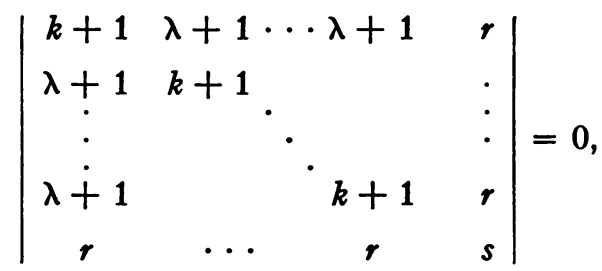

where $r=k+k v / s$ and $s=v+k^{2} v^{2} / t^{2}$. Multiplying row $v+1$ by $-r / s$ and adding to each of the other rows gives $s \cdot \operatorname{det} D=0$, where the matrix $D$ has $k+1-r^{2} / s$ in the main diagonal and $\lambda+1-r^{2} / s$ in all other positions. An evaluation of the determinant of the matrix $D$ implies that

$$
(k-\lambda)^{v-1}\left\{k+1-r^{2} / s+(v-1)\left(\lambda+1-r^{2} / s\right)\right\}=0,
$$

and consequently

$$
t+v-r^{2} v / s=0 .
$$

Since

$$
\frac{r^{2} v}{s}=\frac{k^{2}(t+v)^{2}}{t^{2}+k^{2} v}
$$

it is clear that

$$
(t+v)\left(t^{2}+k^{2} v\right)=k^{2}(t+v)^{2} .
$$


Thus $t^{2}+k^{2} v=k^{2}(t+v)$ and $t=k^{2}$.

COROLLARY. Every element in the arrangement described in the preceding theorem occurs exactly $k$ times and every pair of elements occurs exactly $\lambda$ times.

The corollary is an immediate consequence of the preceding theorem and Theorem 1 derived in [3].

A simple and direct alternative proof of the corollary may be obtained by noting that for $\lambda=k(k-1) /(v-1)$, the equations $s_{1}+\ldots$ $+s_{v}=k v$ and $s_{1}\left(s_{1}-1\right)+\cdots+s_{v}\left(s_{v}-1\right)=\lambda v(v-1)$ imply that $s_{i}=k$ (see [1]). The writer is indebted to Professor Marshall Hall, Jr., for this suggestion.

\section{BIBLIOGRAPHY}

1. R. C. Bose, On the construction of balanced incomplete block designs, Annals of Eugenics vol. 9 (1939) pp. 353-399.

2. R. H. Bruck and H. J. Ryser, The nonexistence of certain finite projective planes, Canadian Journal of Mathematics vol. 1 (1949) pp. 88-93.

3. S. Chowla and H. J. Ryser, Combinatorial problems, Canadian Journal of Mathematics vol. 2 (1950) pp. 93-99.

4. J. A. Todd, A combinatorial problem, Journal of Mathematics and Physics vol. 12 (1933) pp. 321-333.

The Institute for Advanced Study 